\title{
Cirugía torácica en paciente con osteogénesis imperfecta
}

\author{
Thoracic surgery in a patient \\ with osteogenesis imperfecta
}

\author{
Eduardo Leyva-Moraga, ${ }^{*}$ Jesús Martín Ibarra-Celaya, ${ }^{\ddagger}$ Francisco Leyva-Moraga, ${ }^{*}$ Luis Arturo Hurtado-Soto, \\ Graciano Castillo-Ortega," Lilián García-Ballesteros, ${ }^{*}$ Fernando Leyva-Moraga*
}

\author{
*Universidad de Sonora, Hermosillo, Sonora, México. ${ }^{\ddagger}$ Hospital General de Zona No. 2, Hermosillo, México. \\ §Universidad de Monterrey, San Pedro Garza García, Nuevo León, México. \\ "Hospital General del Estado de Sonora, Hermosillo, Sonora, México.
}

\begin{abstract}
RESUMEN. Introducción: La osteogénesis imperfecta es un grupo de anormalidades heterogéneas de la síntesis de colágeno, caracterizada por múltiples fracturas y deformidades. La realización de cirugía torácica en este tipo de pacientes es poco común. Caso clínico: Presentamos el caso de un hombre de 30 años con diagnóstico previo de osteogénesis imperfecta, el cual fue referido a nuestro centro por presentar un empiema loculado hemitórax derecho. Se realizó una toracotomía posterolateral en su hemitórax derecho. En medio de la cirugía pudimos observar una anormalidad anatómica pulmonar que coincide con una cisura accesoria no-ácigos del lóbulo superior. Conclusión: La osteogénesis imperfecta es una entidad clínica rara, sin embargo, el cirujano debe tener en consideración las implicaciones fisiopatológicas durante la cirugía.
\end{abstract}

Palabras clave: Osteogénesis imperfecta, cisura accesoria, empiema.

\section{INTRODUCCIÓN}

La osteogénesis imperfecta $(\mathrm{Ol})$ es un grupo de anormalidades fenotípicas, heterogéneas y hereditarias de la síntesis del colágeno, caracterizada por fragilidad y baja masa ósea. ${ }^{1}$ Comúnmente es de carácter autosómico dominante y su incidencia es de 1 en 15,000-20,000 nacimientos. ${ }^{2}$ La OI está asociada a mutaciones de genes COL1A1 y COL1A2, que codifican las cadenas $\alpha 1(\mathrm{I})$ y $\alpha 2(\mathrm{I})$ del colágeno tipo I, ${ }^{3}$ la proteína más abundante en la matriz extracelular de huesos, tendones y piel. ${ }^{4}$

Sillence y colaboradores clasificaron la OI en cuatro tipos (I, II, III, IV) tomando como referencia manifestacio-

\section{Correspondencia:}

\section{Dr. Francisco Alberto Leyva Moraga}

Universidad de Sonora, Hermosillo, Sonora, México.

Correo electrónico: franciscoleyvam28@gmail.com

Fecha de recepción: 24-VII-2019; aceptado: 12-V-2020.
ABSTRACT. Introduction: Osteogenesis imperfecta is a group of heterogenous abnormalities of the collagen synthesis, characterized by multiple fractures and deformities. Thoracic surgery in patients with osteogenesis imperfecta is not common. Clinical case: We present a case of a 30 years old male with a previous diagnosis of osteogenesis imperfecta who was referred to our center for presenting a loculated empyema on his right hemithorax. A right posterolateral thoracotomy was performed. In the middle of the surgery we visualized an anatomical abnormality that coincides with an accessory fissure of the superior lobe. Conclusion: Osteogenesis imperfecta is a rare clinical entity, however the surgeon must take into consideration the physiopathological implications during the surgery.

Keywords: Osteogenesis imperfecta, accessory fissure, empyema.

nes que van de leves a letales; sin embargo, existen otros 17 subtipos que toman en cuenta patrones radiográficos, histológicos y genéticos. ${ }^{5,6}$

La principal causa de discapacidad funcional y morbimortalidad en pacientes adultos con OI es la disfunción respiratoria, con frecuencia asociada a escoliosis y deformidades de arcos costales que contribuyen al desarrollo de infecciones de vías respiratorias inferiores.?

Presentamos el caso de un paciente con osteogénesis imperfecta, sometido a decorticación pleural por empiema.

\section{PRESENTACIÓN DEL CASO}

Hombre de 30 años de edad, fumador, con antecedente de OI tipo I, referido a nuestro centro por presentar un cuadro clínico de disnea de medianos esfuerzos, acompañado de astenia, adinamia, dolor torácico predominante en hemitórax derecho que aumenta progresivamente en intensidad, tos seca en accesos y picos febriles no cuantificados, de 
dos meses de evolución. La exploración física reveló una disminución de ruidos respiratorios basales en hemitórax derecho, escleróticas azuladas (Figura 1) y manchas acrómicas bien delimitadas en manos y cuello (Figura 2).

Los datos de laboratorio mostraron leucocitosis de 10.7 $10^{3} / \mu \mathrm{L}$, predominio neutrocitario de $8.8110^{3} / \mu \mathrm{L}, \mathrm{DHL} 171$ UI/L y proteínas totales de $6.3 \mathrm{~g} / \mathrm{dL}$. Se realizó telerradiografía de tórax con hallazgos compatibles con derrame pleural loculado derecho, el cual se maneja por sonda endopleural con gasto inicial de $350 \mathrm{~cm}^{3}$. El líquido pleural mostró exudado por leucocitos de 1,300 cel/ $/ \mathrm{mm}^{3}$, predominio de polimorfonucleares de $72 \%$, glucosa de $50 \mathrm{mg} / \mathrm{dL}$, LDH de $1238 \mathrm{UI} / \mathrm{L}$ y proteínas de $5.1 \mathrm{~g} / \mathrm{dL}$, por lo que se llegó al diagnóstico de empiema loculado basal derecho.

Se procedió a realizar lavado y decorticación por medio de toracotomía posterolateral derecha. Considerando el antecedente de OI se tuvo extrema cautela con la movilización del paciente para su traslado y posicionamiento en

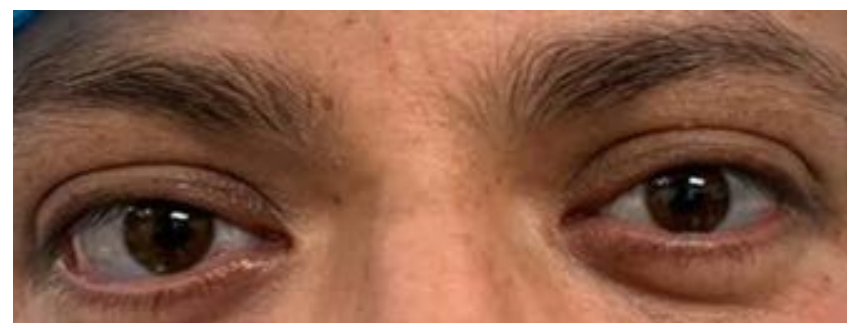

Figura 1: Escleróticas azuladas en paciente con osteogénesis imperfecta.

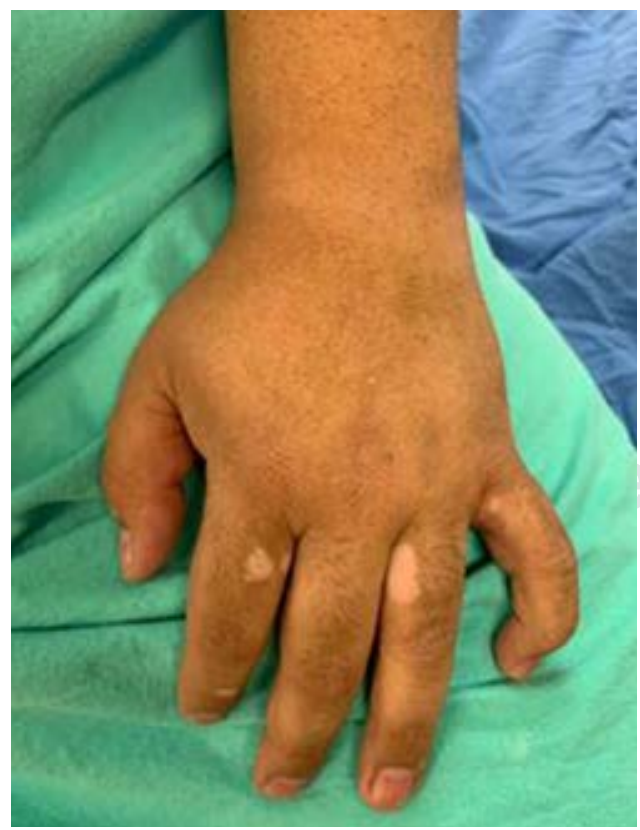

Figura 2: Múltiples manchas acrómicas en dedos de mano izquierda de paciente.

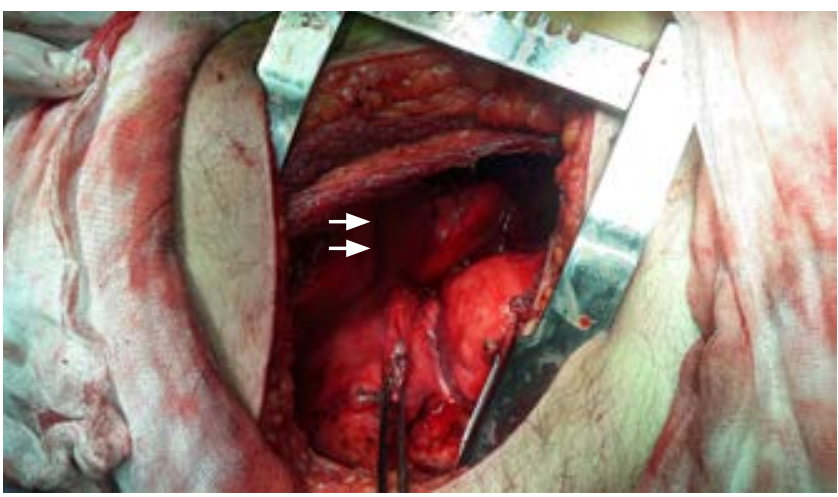

Figura 3: Cisura accesoria incompleta de lóbulo superior por toracotomía (flechas).

la mesa quirúrgica, además de minimizar la movilización cervical para el manejo de vía aérea. La incisión fue realizada a nivel de sexto espacio intercostal, continuando con una disección gentil por planos ingresando a cavidad torácica, donde se observa lóculo posterobasal, conteniendo líquido turbio, atrapamiento pulmonar de predominio en lóbulo inferior por corteza $4 \mathrm{~mm}$, se logra decorticar en un $90 \%$, con adecuada reexpansión pulmonar. La manipulación cuidadosa del parénquima pulmonar fue esencial para la prevención de complicaciones al realizar la técnica quirúrgica. Un hallazgo incidental durante la cirugía fue la presencia de cisura accesoria de lóbulo superior (Figura 3).

El paciente cursó con datos de fuga espiratoria forzada y enfisema subcutáneo leve durante su evolución posquirúrgica, resueltos espontáneamente. Se otorga el alta al séptimo día.

\section{DISCUSIÓN}

Los pacientes con Ol representan un desafío quirúrgico. Aunque el procedimiento no sea directamente en estructuras óseas, es de suma importancia tomar en cuenta la fragilidad de los pacientes con esta condición, sobre todo en la movilización y posicionamiento, así como en la manipulación de la vía aérea.

Las complicaciones hemorrágicas son un aspecto preoperatorio difícil de evaluar, ya que pueden presentar tiempos de coagulación y niveles plaquetarios normales.? Este fenómeno es asociado a una disfunción de las interacciones celulares entre las plaquetas y endotelio debido a la presencia de colágeno anómalo, explicando el aumento de la fragilidad capilar, una ineficiente agregación plaquetaria y a una contracción defectuosa de los pequeños vasos. ${ }^{8,9}$ La incidencia de diátesis hemorrágica en OI es de 10-30\%, lo cual representa un mayor riesgo de presentar sangrado intraoperatorio. El uso de desmopresina intravenosa en pacientes quirúrgicos con $\mathrm{OI}$, ha sido asociado con una 
disminución de uso de paquetes plaquetarios y mejora de la hemostasia. ${ }^{10}$

Los pacientes con OI pueden llegar a presentar dificultades en el manejo de la vía aérea durante la cirugía. Los factores que están relacionados a este evento son la megalocefalia, macroglosia, cuello corto, fragilidad ósea y ruptura de dientes. ${ }^{8,11}$ Se debe tener en cuenta el alto riesgo de presentar fracturas en la columna cervical al manipular el cuello para la intubación o movilización. ${ }^{8,9}$

La OI se ha asociado a un mayor riesgo de desarrollar infecciones de vías respiratorias bajas. Un probable mecanismo causal es la deformidad torácica, la cual puede contribuir a una disminución de la función pulmonar. ${ }^{12}$

La cisura accesoria representa una variación congénita que generalmente ocurre entre segmentos broncopulmonares. ${ }^{13}$ Normalmente estas cisuras pueden ser localizadas por medio de tomografías computarizadas preoperatorias, en este caso no pudo ser valorada por la presencia del empiema. Esta malformación tan peculiar no ha sido descrita en pacientes con OI, la falta de información al respecto nos impide asociar esta variación con la patología en cuestión.

\section{CONCLUSIÓN}

A pesar de que la Ol es una enfermedad rara, es importante que el cirujano conozca las implicaciones fisiopatológicas de la enfermedad y las considere durante todos los tiempos quirúrgicos. El manejo conjunto de un equipo multidisciplinario es indispensable para otorgar la mejor calidad de atención para estos pacientes.

\section{REFERENCIAS}

1. Forlino A, Marini JC. Osteogenesis imperfecta. Lancet 2016;387(10028):1657-1671. https://doi.org/10.1016/s01406736(15)00728-x.

2. Tournis S, Dede AD. Osteogenesis imperfect. A clinical update. Metabolism 2018;80:27-37. https://doi.org/10.1016/j.metabol.2017.06.001.
3. Marini J, Cabral W. Osteogenesis imperfecta. In: Thakker RV, Whyte MP, Eisman JA, Igarashi T, editors. Genetics of bone biology and skeletal disease. 2nd. ed. London Wall: Academic Press, Elsevier; 2018. p. 397-414. doi:10.1016/b978-0-12-804182-6.00023-x.

4. Renaud A, Aucourt J, Weill J, et al. Radiographic features of osteogenesis imperfecta. Insights Imaging 2013;4(4):417-429. https://doi. org/10.1007/s13244-013-0258-4.

5. Morello R. Osteogenesis imperfecta and therapeutics. Matrix Biol 2018;71-72:294-312. https://doi.org/10.1016/j.matbio.2018.03.010-

6. Van Dijk FS, Sillence DO. Osteogenesis imperfecta: clinical diagnosis, nomenclature and severity assessment. Am J Med Genet A 2014;164A(6):1470-1481. https://doi.org/10.1002/ajmg.a.36545.

7. Lafage-Proust MH, Courtois I. The management of osteogenesis imperfecta in adults: State of the art. Joint Bone Spine 2019;86(5):589593. https://doi.org/10.1016/j.jbspin.2019.02.001.

8. Stynowick GA, Tobias JD. Perioperative care of the patient with osteogenesis imperfecta. Orthopedics 2007;30(12):1043-1049. https:// doi.org/10.3928/01477447-20071201-12.

9. Erdoğan MA, Sanli M, Ersoy MO. Anesthesia management in a child with osteogenesis imperfecta and epidural hemorrhage. Braz J Anesthesiol 2013;63(4):366-368. https://doi.org/10.1016/j. bjan.2012.07.007.

10. Keegan MT, Whatcott BD, Harrison BA. Osteogenesis imperfecta, perioperative bleeding, and desmopressin. Anesthesiology 2002;97(4):1011-1013. doi: 10.1097/00000542-200210000-00039.

11. Libman RH. Anesthetic considerations for the patient with osteogenesis imperfecta. Clin Orthop Relat Res 1981;(159):123-125.

12. LoMauro A, Pochintesta S, Romei M, et al. Rib cage deformities alter respiratory muscle action and chest wall function in patients with severe osteogenesis imperfecta. PLoS One 2012;7(4):e35965. https://doi.org/10.1371/journal.pone.0035965.

13. Muttikkal T, Deng C. Non-azygos accessory fissure in right upper lobe associated with superior and inferior accessory fissures in right lower Lobe. J Clin Imaging Sci 2012;2:79. https://doi.org/10.4103/21567514.105133.

Conflicto de intereses: Los autores certifican no estar involucrados en alguna organización con interés financiero o no financiero con respecto a los temas discutidos en el manuscrito. 